\title{
Tapered polysilicon core fibers for nonlinear photonics
}

\author{
Fariza H. Suhailin, ${ }^{1,2}$ LI Shen, ${ }^{1}$ Noel Healy, ${ }^{1,3}$ LIMIN XIAO, ${ }^{1}$ MaXWell Jones, ${ }^{4}$ \\ ThOMAS HaWkins, ${ }^{4}$ JOHn Ballato, ${ }^{4}$ URSUla J. GibSON, ${ }^{5}$ AND ANNA C. \\ PEACOCK $^{1, *}$
}

\author{
${ }^{1}$ Optoelectronics Research Centre, University of Southampton, SO17 1BJ, UK \\ ${ }^{2}$ School of Fundamental Science, Universiti Malaysia Terengganu, 21300 Kuala Terengganu, Malaysia \\ ${ }^{3}$ Emerging Technology and Materials Group, Newcastle University, NE1 7RU, UK \\ ${ }^{4}$ COMSET, School of Materials Science and Engineering, Clemson University, SC 29634, USA \\ ${ }^{5}$ Department of Physics, Norwegian University of Science and Technology, 7491 Trondheim, Norway \\ *Corresponding author: acp@soton.ac.uk
}

Received XX Month XXXX; revised XX Month, XXXX; accepted XX Month XXXX; posted XX Month XXXX (Doc. ID XXXXX); published XX Month XXXX

\begin{abstract}
We propose and demonstrate a novel approach to obtaining small core polysilicon waveguides from the silicon fiber platform. The fibers were fabricated via a conventional drawing tower method and then subsequently tapered down to achieve silicon core diameters of $\sim 1 \mu \mathrm{m}$, the smallest optical cores for this class of fiber to date. Characterization of the material properties have shown that the taper process helps to improve the local crystallinity of the silicon core, resulting in a significant reduction in the material loss. By exploiting the combination of small cores and low losses, these tapered fibers have enabled the first observation of nonlinear transmission within a polycrystalline silicon waveguide of any type. As the fiber drawing method is highly scalable, it opens a route for the development of low cost and flexible nonlinear silicon photonic systems. (C) 2015 Optical Society of America
\end{abstract}

OCIS codes: (060.2280) Fiber design and fabrication; (060.2290) Fiber materials; (190.4370) Nonlinear optics, fibers; (060.6000) Semiconductor materials.

http://dx.doi.org/10.1364/OL.99.099999

Over the past few decades, silicon photonics has become one of the largest growing research areas of our time. Major breakthroughs include the development of devices capable of light generation [1],

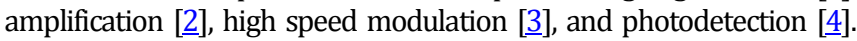
These advances have principally been enabled by the excellent optical and electronic properties of the well-established single crystal siliconon-insulator (SOI) platforms. However, a significant drawback of SOI is that, owing to the strict requirements on the single crystal material growth, it is somewhat inflexible, which can hinder its integration either with other chip-based components or the optical fibers that are used to link the systems together. More recently there has been growing interest in waveguides fabricated from polysilicon (p-Si) materials which are much cheaper and easier to produce, so that they can be incorporated into a wider range of geometries [ $[5]$ and architectures [6]. To date, devices fabricated from p-Si materials have been demonstrated for multilayer coupling [7]], high speed modulators [8], and photodetection [9]. However, as yet, there have been no reports of nonlinear optical device development in this material system, most likely due to the high reported propagation losses in waveguides with small, few microns to hundreds of nanometers, sized dimensions [6, 7].

There are two main factors that contribute to the losses in p-Si waveguides; the first is associated with the bulk material quality, i.e., scattering and absorption at the grain boundaries, and the second is due to surface roughness, which is typically enhanced by the grain structure of the material. Recent advances in the development of planar p-Si platforms have shown that it is possible to reduce the bulk absorption losses to below $3 \mathrm{~dB} / \mathrm{cm}$ in waveguides with large core sizes (widths greater than $5 \mu \mathrm{m}$ ), though sidewall roughness still limits

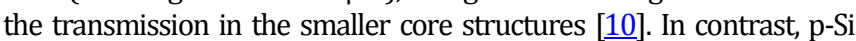
waveguides that are fabricated within the optical fiber platform have been shown to have near atomically smooth surfaces as the polycrystalline material is confined within the pristine silica glass cladding [11]. As a result, p-Si fibers can be fabricated to have negligible surface scattering losses for the micro/nanometer sized core diameters required for the observation of nonlinear processes. Though, compared to its planar counterparts, the silicon fiber technology is still in its infancy, significant progress has been made towards reducing the bulk material losses, with a value of $\sim 5 \mathrm{~dB} / \mathrm{cm}$ recently reported for a $\mathrm{p}$-Si fiber with a core diameter of $\sim 2 \mu \mathrm{m}[\underline{12}]$. As a result, these fibers are attracting increased interest for their ability to develop the semiconductor waveguide materials directly within the fiber geometry.

In this letter, we investigate a novel route to obtaining high optical quality, small core p-Si waveguides starting from the silicon fiber platform. The approach is based on a modified tapering procedure in which the pre-fabricated fiber is heated and drawn within a capillary sleeve to reduce the core size, whilst retaining a robust and strong cladding. Significantly, previous work conducted on germanium core 
fibers has indicated that such tapering methods should also help improve the crystallinity of the core by enhancing the grain growth and aligning the crystal orientation with respect to the fiber axis [13]. By applying this method to fibers with a silicon core material, we provide confirmation of the enhanced transmission properties that can be obtained via this route, and examine the role of the tapering ratio. The lowest loss of $3.5 \mathrm{~dB} / \mathrm{cm}$ was obtained for a fiber with a diameter of only $\sim 1 \mu \mathrm{m}$, which is the smallest core size reported for optical transmission in a crystalline silicon fiber to date. Significantly, this combination of small core size and low loss has allowed for the first characterization of nonlinear propagation in a p-Si waveguide, either fiber or planar-based, with the estimated material parameters being in good agreement with values reported for single crystal silicon. We anticipate that these tapered $\mathrm{p}$-Si fibers will be of interest for a range of nonlinear signal processing applications [14].

The silicon fibers used in this work were fabricated via the molten core drawing method described in [15]. Briefly, the inner silica capillary preform was first coated with a calcium oxide $(\mathrm{CaO})$ interface modifier before a silicon rod was inserted into the core. The $\mathrm{CaO}$ layer has two main purposes; firstly, it serves as a 'sink for the impurities and secondly, it helps to reduce the thermal strain during the drawing process. The fiber was subsequently drawn using a conventional tower at a temperature of $\sim 1950{ }^{\circ} \mathrm{C}$, where the silica cladding glass is softened. As this temperature is above the melting temperature of silicon, $\sim 1414^{\circ} \mathrm{C}$, it allows the molten core material to take on the form of the smooth glass cladding as it is drawn into the optical fiber. The final fabricated fiber had an outer diameter of OD 130 $\mu \mathrm{m}$ and an inner core diameter of ID $\sim 10 \mu \mathrm{m}$, which was polycrystalline in nature [16]. We note that controlling the draw parameters to directly obtain fibers with smaller dimensions, without (Rayleigh-Plateau instability) break-up of the core, is an ongoing technical challenge. Thus tapering the fiber, post-draw, provides a convenient route to obtaining the core sizes desired for many optical applications.

(a)

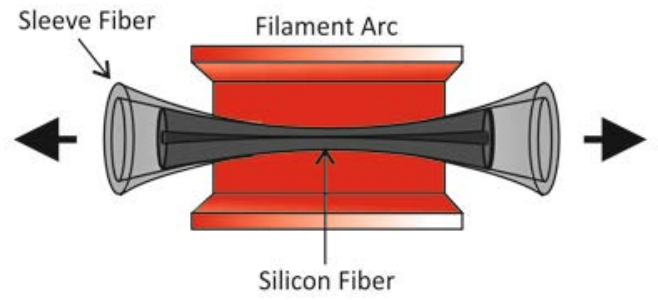

(b)

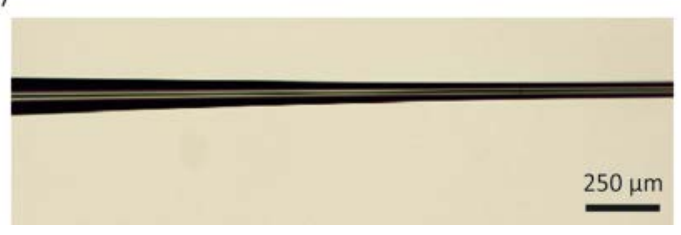

Fig. 1. (a) Schematic of the tapering procedure in which the silicon fiber is sleeved into a longer capillary. The filament heats the combined structure, which is drawn in the direction of the arrows. (b) Longitudinal image of a tapered silicon core fiber.

In order to reach core sizes of a few micrometers in diameter, we have developed a new tapering procedure in which the as-drawn fibers are first sleeved inside thicker capillary (ID/OD diameters of 150/200 $\mu \mathrm{m}$ ), as illustrated in Fig. 1(a). The sleeve offers two key benefits in that it not only helps to strengthen the silicon fibers as their dimensions are reduced, ensuring their integrity is preserved, but it also reduces the length of silicon fiber required for processing from $\sim 20 \mathrm{~cm}$ down to $\sim 1 \mathrm{~cm}$. The sleeved fibers are then tapered using a Vytran tapering rig (GPX-3300). By carefully setting the taper parameters, the pulling speed and the filament power, both the silica sleeve and silicon fiber cladding soften, and fuse, while at the same time the silicon core melts so that it flows through the reshaped cladding, before recrystallizing as it cools. The result is a smooth, continuous, tapering from the untapered fiber down to the waist, as shown in Fig. 1(b). This method is highly versatile and fibers have been fabricated with taper ratios of 1:2 (fiber A), 1:3 (fiber B) and 1:10 (fiber C), as illustrated in Fig. 2. In all cases the cladding diameters change proportionally to the core, and the total taper length is typically $\sim 2 \mathrm{~cm}$, of which there is a constant waist region of $\sim 1 \mathrm{~cm}$. By maintaining a slowly varying transition region between the untapered fiber and the uniform taper waist, coupling between the modes supported by the micron sized cores and/or the radiation modes of the cladding will be minimal.
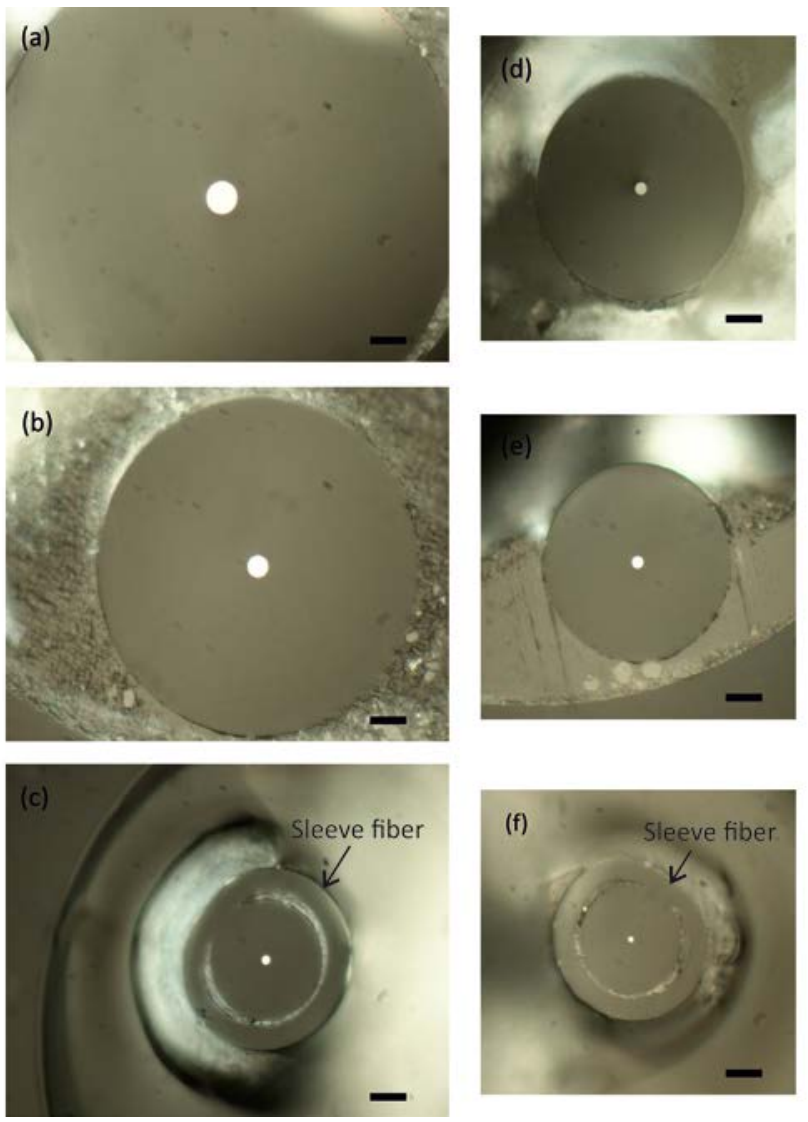

Fig. 2. Microscope images of the polished fiber cross sections. Input facets for (a) tapered fiber A, (b) fiber B, and (c) fiber C. Tapered waists for (d) fiber A, (e) fiber B, and (f) fiber C. All scale bars are $10 \mu \mathrm{m}$.

To study the effects of the tapering on the quality of the polycrystalline core material, micro-Raman measurements were performed at the taper waist for the three fibers and compared with the as-drawn material. A $100 \times$ objective lens was used to focus $\sim 3 \mathrm{~mW}$ of $532 \mathrm{~nm}$ laser power onto the silicon core through the transparent silica cladding, and the backscattered radiation was recorded on a thermoelectrically cooled CCD detector. The results for each of the tapered waists are shown in Fig. 3, together with a typical spectrum for the untapered fiber. A Voigt function (dashed curves) was used to fit the spectra to deconvolve the Lorentzian peak of the material from the 
instrument broadening, which is Gaussian shaped, with the extracted Lorentzian widths given in the legend. For comparison the silicon reference has a width of $\Gamma=2.7 \mathrm{~cm}^{-1}$. The estimated Raman widths of the measured fibers indicate that there has been an improvement of the polycrystalline core material during the tapering process, in accordance with the results of [13]. Furthermore, the improvement appears to be more pronounced for the larger taper ratios, with the width of fiber $\mathrm{C}$ approaching that of the single crystal reference. This trend is not unexpected as the smaller cores will experience a higher cooling rate, which suppresses the number of nucleation sites $[\underline{17}, \underline{18}]$. We note that the slight downshifting in the position of Raman peaks in the tapered fibers from the expected value of $520 \mathrm{~cm}^{-1}$ is most likely due to some residual strain associated with differences in the thermal expansion of the core/cladding materials [19]. A detailed characterization of the crystalline quality for the different tapered core sizes is still underway, and will be the subject of a separate paper.

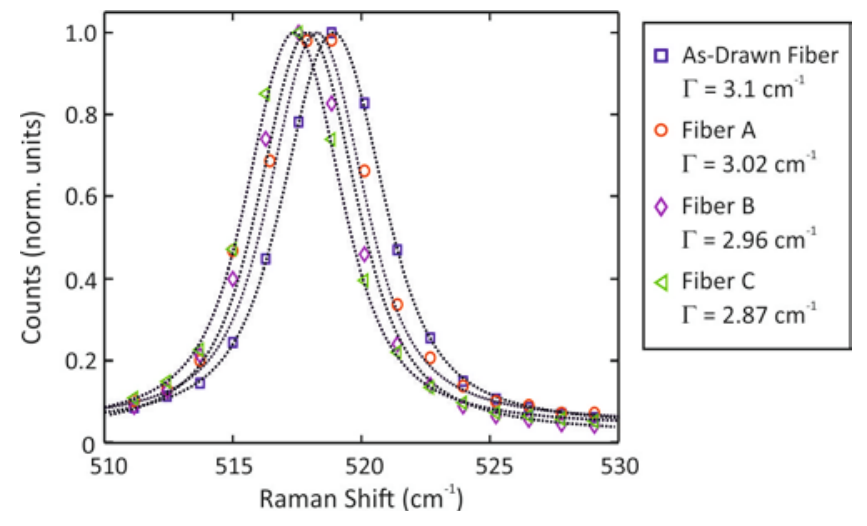

Fig. 3. Raman spectra for the p-Si core material taken for the as-drawn fiber and at the tapered waists of fibers A, B and C. The dash curves are the Voigt fits and the Lorentzian linewidths are given in the legend.

Linear transmission measurements were then performed to investigate the optical quality of the tapered core materials. To ensure efficient coupling, the end facets were prepared using a standard polishing procedure, as described in [19]. The polished cross sections for the three fibers were already presented in Fig. 2, where the left hand images show representative dimensions for the starting input/output facets of (a) fiber A, with D $9.4 \mu \mathrm{m}$, (b) fiber B, with D $6.2 \mu \mathrm{m}$ and (c) fiber $\mathrm{C}$, with $\mathrm{D} \sim 2.4 \mu \mathrm{m}$. Linear transmission measurements were performed using a continuous wave $1550 \mathrm{~nm}$ laser diode $(\sim 6 \mathrm{~mW})$, which was launched into the fibers using either a $40 \times$ microscope objective lens for fibers $A$ and $B$, or a $63 \times$ objective for fiber C. A second $25 \times$ microscope objective lens was used to capture the output light and focus it onto a power meter. As these micron sized core fibers support multiple modes, to ensure that the light was preferentially coupled into the fundamental mode, 90/10 beam splitters were placed either side of the fiber under test and the input and output beams were monitored via two CCD cameras [20]. The losses were measured using a cut-back method, whereby lengths of a few millimeters were repeatedly polished off the end of the fibers until the tapered waists were reached, shown in the right hand images of Fig. 2. The waist diameters for the three fibers are (d) $D_{w} \sim 4.8 \mu \mathrm{m}$, (e) $\mathrm{D}_{\mathrm{w}} \sim 3.5 \mu \mathrm{m}$ and (f) $\mathrm{D}_{\mathrm{w}} \sim 0.94 \mu \mathrm{m}$. These images confirm that the tapered fibers maintain a solid and circular core, as well as a welldefined core/clad region.

Table 1 summarizes the waveguide losses for all the p-Si fibers, including values for the three tapered fibers as well as the unprocessed fiber. We note that these values represent the average loss of the fundamental mode from the cut-backs, but that no significant change in
Table 1. Measured linear loss values for the tapered p-Si fibers.

\begin{tabular}{llll}
\hline \hline Fiber & Taper Ratio & $\begin{array}{l}\mathrm{D}_{\mathrm{w}} \\
(\mu \mathrm{m})\end{array}$ & $\begin{array}{l}\text { Loss } \\
(\mathrm{dB} / \mathrm{cm})\end{array}$ \\
\hline Original & Untapered & 10.0 & 12.0 \\
$\mathrm{~A}$ & $1: 2$ & 4.80 & 8.0 \\
$\mathrm{~B}$ & $1: 3$ & 3.46 & 5.6 \\
$\mathrm{C}$ & $1: 10$ & 0.94 & 3.5 \\
\hline \hline
\end{tabular}

the loss was recorded for different sections (i.e., diameters) of the fibers, indicating that the dominant contribution comes from the bulk core material. The high loss of the untapered fiber was expected as previous investigations have revealed that when drawing silicon fiber, the average grain size decreases as a function of decreasing core diameter, so that a reasonable estimate of the grain sizes in our $10 \mu \mathrm{m}$ core fiber would be $<1 \mathrm{~mm}$ [17]. However, in contrast, the losses are found to reduce dramatically in the fibers tapered to a high ratio, with the lowest loss of $3.5 \mathrm{~dB} / \mathrm{cm}$ obtained in the fiber with a submicron waist. These results, which are in agreement with the Raman linewidths presented in Fig. 3, provide further verification that the taper process acts to improve the local crystallinity and reduce the defects in the bulk material. To investigate the reproducibility of the tapering process, additional small core fibers were fabricated with waist diameters in the range 1-2 $\mu \mathrm{m}$. These fibers all recorded similar Raman linewidths and losses within the region of $3-4 \mathrm{~dB} / \mathrm{cm}$, confirmation that the crystallinity of the core material has been improved by a comparable amount. To the best of our knowledge, the losses in these tapered fibers are the lowest values reported for $\mathrm{p}-\mathrm{Si}$ waveguides with core sizes of only a few microns $[\underline{6}, \underline{10}, \underline{12}]$. It is worth recalling that as the core/cladding interface in the fibers is ultrasmooth, we attribute the residual losses to defects and impurities that remain in the bulk material, and work is ongoing to reduce these to an even lower level. Nevertheless, the relatively low losses measured for the smallest core fiber $\mathrm{C}$ present a unique opportunity to investigate the nonlinear properties of this material for the first time.

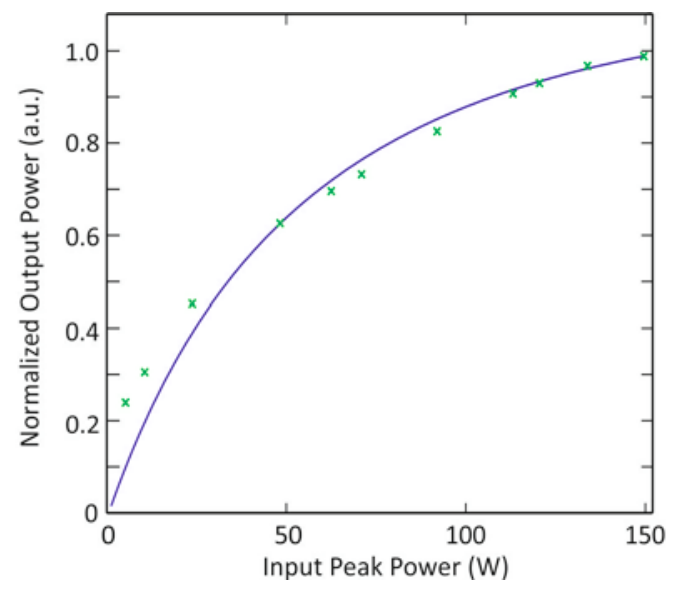

Fig. 4. Nonlinear absorption as a function of coupled input peak power. Solid curve is the simulated fit to estimate the TPA parameter.

Nonlinear transmission in silicon waveguides is governed by two key processes; the two-photon absorption (TPA) and Kerr nonlinear refraction. As TPA manifests as an intensity dependent absorption, the TPA parameter $\left(\beta_{\text {TPA }}\right)$ can be determined simply by monitoring the output power as a function of the coupled input power. Subsequently, the size of the nonlinear refractive index $\left(n_{2}\right)$ can be estimated by mapping the spectral broadening due to self-phase modulation (SPM). To undertake these measurements we employed a high power fiber 
laser delivering $720 \mathrm{fs}$ (FHWM) pulses, with a $40 \mathrm{MHz}$ repetition rate, at a wavelength of $1540 \mathrm{~nm}$. The light from this source was free space launched into the fundamental mode of fiber $\mathrm{C}$ using a $63 \times$ objective lens, with a second $25 \times$ microscope objective lens to capture the output, which was directed either to a power meter or an optical spectrum analyzer. The results of the nonlinear absorption measurements are shown in Fig. 4, while the spectral broadening is plotted in Fig. 5. We note that the slight oscillations on the spectra in Fig. 5 are most likely due to a small amount of coupling to higher order modes, though from the results in [20] we estimate this to be no more than a few percent, evidence that the propagating light has primarily remained in the fundamental mode. In both cases the experimental data is fit using a standard nonlinear Schrödinger equation, as described in [21], with the remaining material parameters estimated from the single crystal values. From these results we determine the best fit values of $\beta_{\text {TPA }} \sim 0.7 \mathrm{~cm} / \mathrm{GW}$ and $n_{2} \sim 0.5 \times 10^{-14} \mathrm{~cm}^{2} / \mathrm{W}$. Significantly, both these values are within the range previously reported for single crystal silicon $[22,23]$, which is as we would expect for a high optical quality $\mathrm{p}-\mathrm{Si}$ material. Thus these measurements provide a useful benchmark from which to develop future nonlinear optical devices in the p-Si waveguides, either in fiber or planar form.

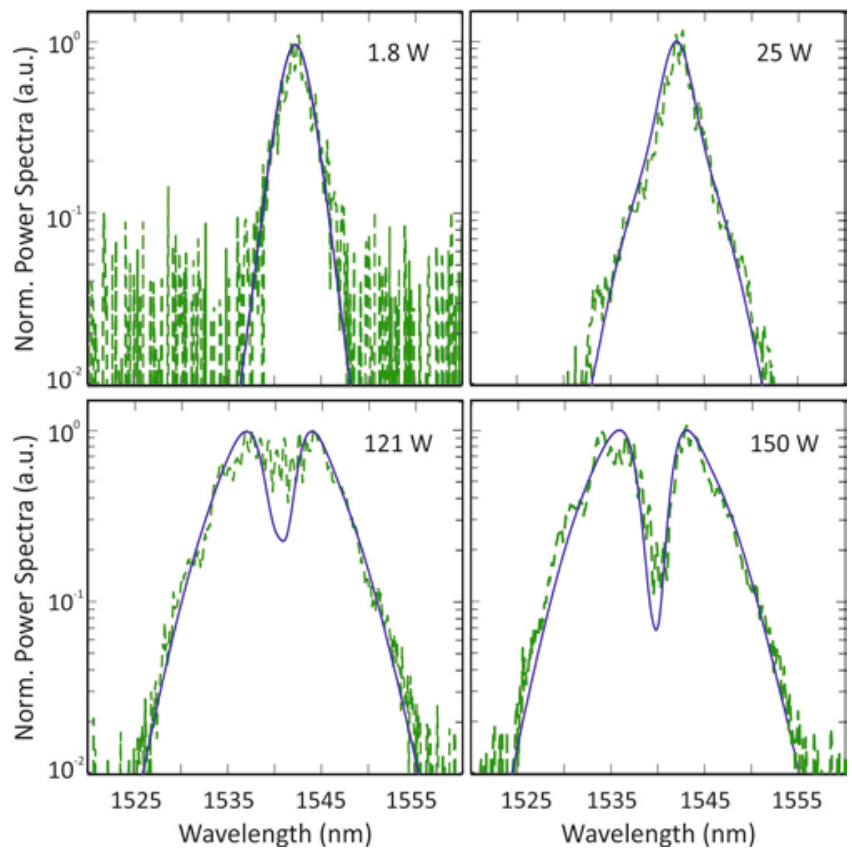

Fig. 5. Spectral evolution as a function of coupled peak power (see legends) for fiber $\mathrm{C}$ (dash curves). Solid curves are simulated fits to estimate the nonlinear refractive index.

In conclusion, we have proposed and demonstrated a novel approach to fabricating low loss, small core p-Si waveguides from the silicon optical fiber platform. Significantly, the tapering process not only provides a mechanism through which to improve the material quality, but also allows for control over the core size, which could in turn be used to tailor the dispersion and nonlinear properties of the waveguides [14]. By reducing the loss to $\sim 3 \mathrm{~dB} / \mathrm{cm}$, these waveguides have enabled the first observation the nonlinear transmission in the $\mathrm{p}$ Si material, from which we could verify that the nonlinear parameters are in agreement with those of single crystal silicon. Thus we can expect that with continued reduction in the loss values, $\mathrm{p}-\mathrm{Si}$ waveguides will emerge as desirable components for the development of low cost and flexible nonlinear optical systems.

\section{Sample dataset citation}

The data for this work is accessible through the University of Southampton Institutional Research Repository (DOI:10.5258/SOTON/385267).

Funding. EPSRC (EP/J004863/1); NORFAB; Norwegian Discovery Fund; Malaysian Education Ministry (MOE) and Universiti Malaysia Terengganu (UMT).

Acknowledgment. We thank Yohann Franz for assistance with the Raman measurements.

\section{References}

1. H. Rong, R. Jones, A. Liu, O. Cohen, D. Hak, A. Fang, and M. Paniccia, Nature 433, 725 (2005).

2. R. Claps, D. Dimitropoulos, V. Raghunathan, Y. Han, and B. Jalali, Opt. Express 11, 1731 (2003).

3. G. T. Reed, G. Mashanovich, F. Y. Gardes, and D. J. Thomson, Nat. Photon. 4, 518 (2010).

4. I. Goykhman, B. Desiatov, J. Khurgin, J. Shappir, and U. Levy, Opt. Express 20, 28594 (2012).

5. J. Ballato, T. Hawkins, P. Foy, R. Stolen, B. Kokuoz, M. Ellison, C. McMillen, J. Reppert, A. M. Rao, M. Daw, S. R. Sharma, R. Shori, O. Stafsudd, R. R. Rice, and D. R. Powers, Opt. Express 16, 18675 (2008).

6. J. S. Orcutt, S. D. Tang, S. Kramer, K. Mehta, H. Li, V. Stojanović, and R. J. Ram, Opt. Express 20, 7243 (2012).

7. Q. Fang, J. F. Song, S. H. Tao, M. B. Yu, G. Q. Lo, and D. L. Kwong, Opt. Express 16, 6425 (2008).

8. K. Preston, P. Dong, B. Schmidt, and M. Lipson, Appl. Phys. Lett. 92, 151104 (2008).

9. K. Preston, Y. H. D. Lee, M. Zhang, and M. Lipson, Opt. Lett. 36, 52 (2011).

10. D. Kwong, J. Covey, A. Hosseini, Y. Zhang, X. Xu, and R. T. Chen, Opt. Express 20, 21722 (2012).

11. N. Healy, L. Lagonigro, J. R. Sparks, S. Boden, P. J. A. Sazio, J. V. Badding, and A. C. Peacock, Opt Lett 36, 2480 (2011).

12. N. Healy, S. Mailis, N. M. Bulgakova, P. J. A. Sazio, T. D. Day, J. R. Sparks, H. Y. Cheng, J. V. Badding, and A. C. Peacock, Nat. Mater. 13, 1122 (2014).

13. C. McMillen, G. Brambilla, S. Morris, T. Hawkins, P. Foy, N. Broderick, E. Koukharenko, R. Rice, and J. Ballato, Opt. Mater. 35, 93 (2012).

14. A. C. Peacock, Opt. Lett. 35, 3697 (2010).

15. F. A. Martinsen, B. K. Smeltzer, M. Nord, T. Hawkins, J. Ballato, and U. J. Gibson, Sci. Rep. 46283 (2014)

16. E. F. Nordstrand, A. N. Dibbs, A. J. Eraker, and U. J. Gibson, Opt. Mater. Express 3, 651 (2013).

17. N. Healy, J. R. Sparks, P. J. Sazio, J. V. Badding, and A. C. Peacock, Opt. Express 18, 7596 (2010).

18. B. L. Scott and G. R. Pickrell, J. Cryst. Growth 371, 134 (2013).

19. L. Lagonigro, N. Healy, J. R. Sparks, N. F. Baril, P. J. A. Sazio, J. V. Badding, and A. C. Peacock, Appl. Phys. Lett. 96, 0411051 (2010).

20. A. C. Peacock, P. Mehta, P. Horak, and N. Healy, Opt. Lett. 37, 3351 (2012).

21. P. Mehta, N. Healy, N. F. Baril, P. J. A. Sazio, J. V. Badding, and A. C. Peacock, Opt. Express 18, 16826 (2010).

22. M. Dinu, F. Quochi, and H. Garcia, Appl.Phys. Lett. 82, 2954 (2003).

23. A. D. Bristow, N. Rotenberg, and H. M. van Driel, Appl. Phys. Lett. 90, 191104 (2007). 


\section{Fifth page for references}

1. H. Rong, R. Jones, A. Liu, O. Cohen, D. Hak, A. Fang, and M. Paniccia, "A continuous-wave Raman silicon laser," Nature 433, 725-728 (2005).

2. R. Claps, D. Dimitropoulos, V. Raghunathan, Y. Han, and B. Jalali, "Observation of stimulated Raman amplification in silicon waveguides," Opt. Express 11, 1731-1739 (2003).

3. G. T. Reed, G. Mashanovich, F. Y. Gardes, and D. J. Thomson, "Silicon optical modulators," Nat. Photon. 4, 518-526 (2010)

4. I. Goykhman, B. Desiatov, J. Khurgin, J. Shappir, and U. Levy, "Waveguide based compact silicon Schottky photodetector with enhanced responsivity in the telecom spectral band," Opt. Express 20, 28594-28602 (2012).

5. J. Ballato, T. Hawkins, P. Foy, R. Stolen, B. Kokuoz, M. Ellison, C. McMillen, J. Reppert, A. M. Rao, M. Daw, S. R. Sharma, R. Shori, O. Stafsudd, R. R. Rice, and D. R. Powers, "Silicon optical fiber," Opt. Express 16, 1867518683 (2008).

6. J. S. Orcutt, S. D. Tang, S. Kramer, K. Mehta, H. Li, V. Stojanović, and R. J. Ram, "Low-loss polysilicon waveguides fabricated in an emulated highvolume electronics process," Opt. Express 20, 7243-7254 (2012).

7. Q. Fang, J. F. Song, S. H. Tao, M. B. Yu, G. Q. Lo, and D. L. Kwong, "Low loss $(\sim 6.45 \mathrm{~dB} / \mathrm{cm})$ sub-micronpolycrystalline silicon waveguide integrated with efficient SiON waveguide coupler," Opt. Express 16, 6425-6432 (2008).

8. K. Preston, P. Dong, B. Schmidt, and M. Lipson, "High-speed all-optical modulation using polycrystalline silicon microring resonators," Appl. Phys. Lett. 92, 151104 (2008).

9. K. Preston, Y. H. D. Lee, M. Zhang, and M. Lipson, "Waveguide-integrated telecom-wavelength photodiode in deposited silicon," Opt. Lett. 36, 52-54 (2011).

10. D. Kwong, J. Covey, A. Hosseini, Y. Zhang, X. Xu, and R. T. Chen, "Ultralowloss polycrystalline silicon waveguides and high uniformity $1 \times 12 \mathrm{MMI}$ fanout for 3D photonic integration," Opt. Express 20, 21722-21728 (2012).

11. N. Healy, L. Lagonigro, J. R. Sparks, S. Boden, P. J. A. Sazio, J. V. Badding, and A. C. Peacock, "Polycrystalline silicon optical fibers with atomically smooth surfaces," Opt. Lett. 36, 2480-2482 (2011).

12. N. Healy, S. Mailis, N. M. Bulgakova, P. J. A. Sazio, T. D. Day, J. R. Sparks, H. Y. Cheng, J. V. Badding, and A. C. Peacock, "Extreme electronic bandgap modification in laser-crystallized silicon optical fibres," Nat. Mater. 13, 1122-1127 (2014).

13. C. McMillen, G. Brambilla, S. Morris, T. Hawkins, P. Foy, N. Broderick, E. Koukharenko, R. Rice, and J. Ballato, "On crystallographic orientation in crystal core optical fibers II: Effects of tapering," Opt. Mater. 35, 93-96 (2012).

14. A. C. Peacock, "Soliton propagation in tapered silicon core fibers," Opt. Lett. 35, 3697-3699 (2010).

15. F. A. Martinsen, B. K. Smeltzer, M. Nord, T. Hawkins, J. Ballato, and U. J. Gibson, "Silicon-core glass fibres as microwire radial-junction solar cells," Sci. Rep. 46283 (2014).

16. E. F. Nordstrand, A. N. Dibbs, A. J. Eraker, and U. J. Gibson, "Alkaline oxide interface modifiers for silicon fiber production," Opt. Mater. Express 3, 651-657 (2013).

17. N. Healy, J. R. Sparks, P. J. Sazio, J. V. Badding, and A. C. Peacock, "Tapered silicon optical fibers," Opt. Express 18, 7596-7601 (2010).

18. B. L. Scott, and G. R. Pickrell, "Silicon optical fiber diameter dependent grain size," J. Cryst. Growth 371, 134-141 (2013).

19. L. Lagonigro, N. Healy, J. R. Sparks, N. F. Baril, P. J. A. Sazio, J. V. Badding, and A. C. Peacock, "Low loss silicon fibers for photonics applications," Appl. Phys. Lett. 96, 0411051-0411053 (2010).

20.A. C. Peacock, P. Mehta, P. Horak, and N. Healy, "Nonlinear pulse dynamics in multimode silicon core optical fibers," Opt. Lett. 37, 3351 (2012).
21. P. Mehta, N. Healy, N. F. Baril, P. J. A. Sazio, J. V. Badding, and A. C. Peacock, "Nonlinear transmission properties of hydrogenated amorphous silicon core optical fibers," Opt. Express 18, 16826-16831 (2010).

22. M. Dinu, F. Quochi, and H. Garcia, "Third-order nonlinearities in silicon at telecom wavelengths," Appl. Phys. Lett. 82, 2954-2956 (2003).

23. A. D. Bristow, N. Rotenberg, and H. M. van Driel, "Two-photon absorption and Kerr coefficients of silicon for 850-2200nm," Appl. Phys. Lett. 90, 191104 (2007). 